\title{
Theoretical Analysis of Lewis Basicity \\ Based on Local Electron-Donating Ability. \\ Origin of Basic Strength of Cyclic Amines
}

\author{
Tomohiko Ohwada*,$\perp$ Hajime Hirao, ${ }^{\perp}, \$$ Atsushi Ogawa\% \\ ${ }^{\perp}$ Graduate School of Pharmaceutical Sciences, The University of Tokyo, Tokyo 113-0033, \\ Japan, and ${ }^{\%}$ Division of Molecular Engineering, Kyoto University, Kyoto 606-8501, \\ Japan. \\ E-mail: ohwada@mol.f.u-tokyo.ac.jp
}

\section{Contents}

Figure S1 B3LYP/6-31G* Optimized Structures of N-Unsubstituted Cyclic Amines and Their N-Protonated Ions S2

Figure S2 B3LYP/6-31G* Optimized Structures of N-Methyl-Substituted Cyclic Amines and Their N-Protonated Ions S3

Table S1 - Table S15 The Coordinates of Atoms of Neutral Amines S4- S12.

Table S16 - Table S28 The Coordinates of Atoms of N-protonated Amines S12S19.

Table S29 Redistribution Charge upon N-Protonation of the Amines. Natural Population Analysis (B3LYP/6-31G* level) of the N-H atoms of the N-protonataed Amines and the N Atom of the Neutral Amine based on B3LYP6-31G* Optimized Structures. S20

Table S30 The pyramidalization angle $\left(\beta_{1}, \beta_{2}\right.$, and $\beta_{3}$.see Scheme 1$)$ of inner and outer components of the RHO of the amine nitrogen atom (which represents the reactive lone-pair electrons). $\quad \mathbf{S 2 1}$

Table S31 The average values of $\beta_{1}, \beta_{2}$, and $\beta_{3}$ of the inner and outer RHOs of $\mathrm{N}, \mathrm{N}$-dimethylamine upon changes of the bond angle $(\alpha)$. Comparison with the $\beta$ values. $\mathbf{S 2 2}$

Table S32 Summary of the Percentage s, p, and d Characters of the Nitrogen Atom Component of the RHO $\mathbf{S 2 3}$ 

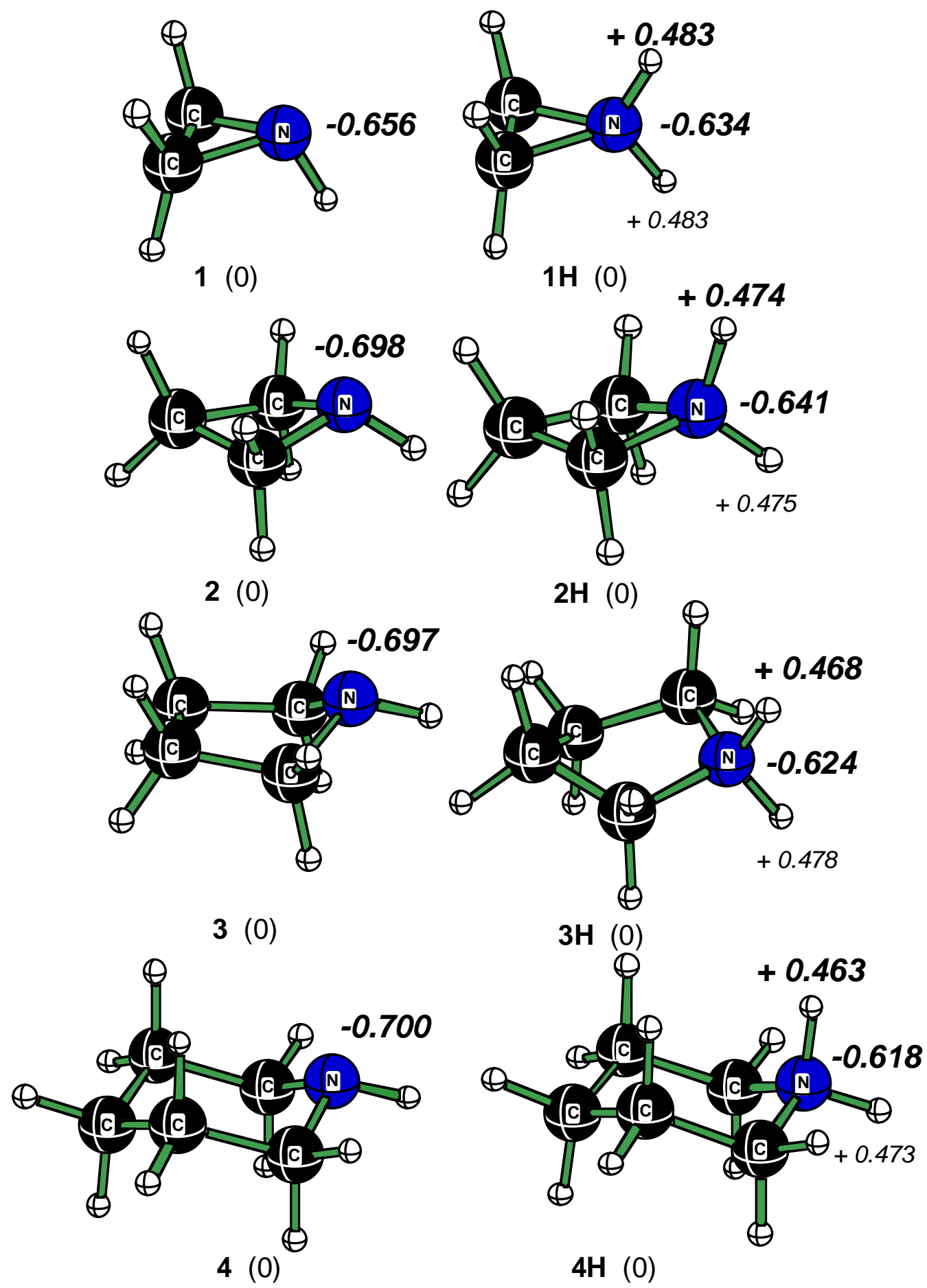

Figure S1 B3LYP/6-31G* Optimized Structures of N-Unsubstituted Cyclic Amines and Their N-Protonated lons

The imaginary frequency number is shown in parentheses. The natural population charges are shown italic. 

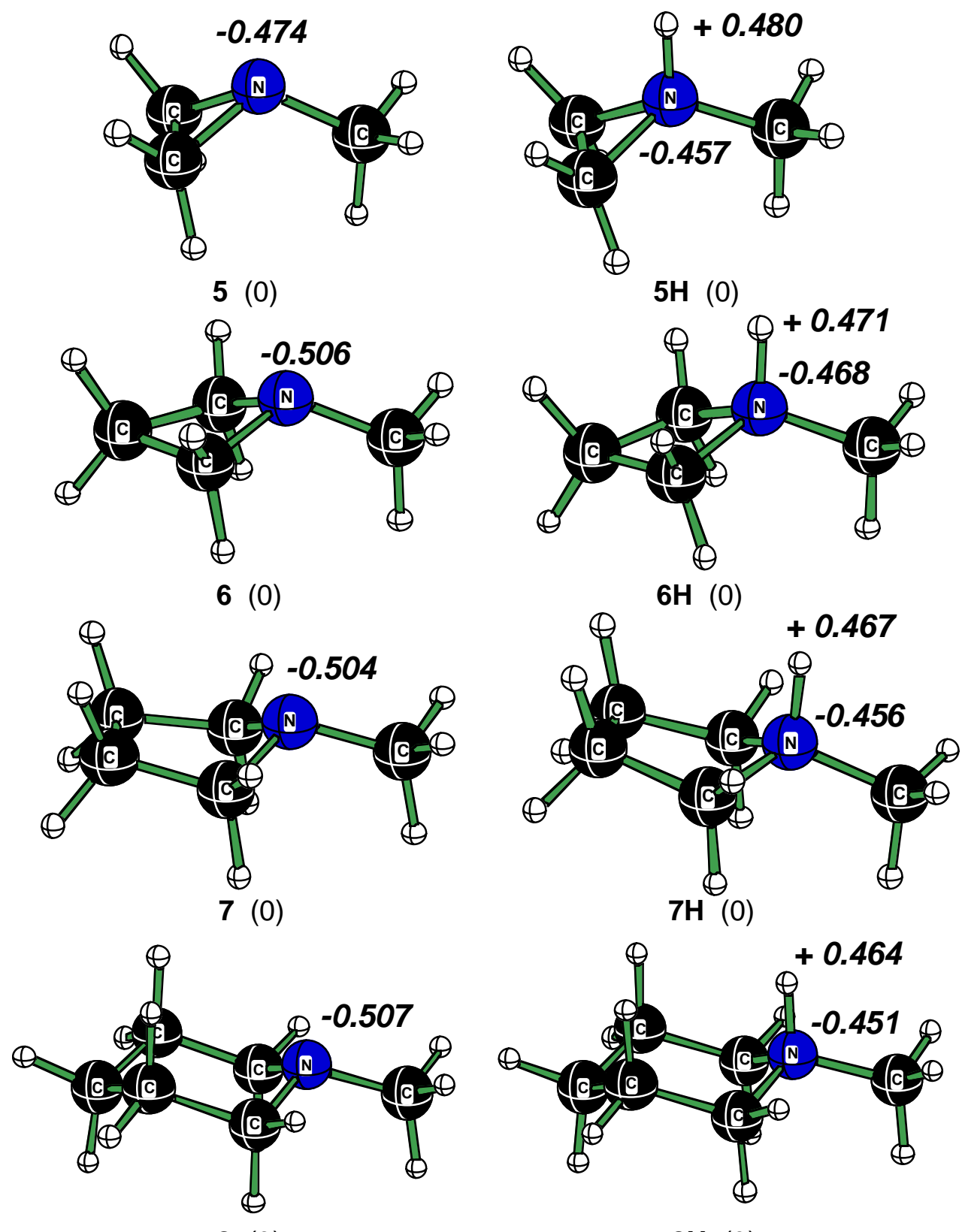

$8(0)$

8H (0)

Figure S2 B3LYP/6-31G* Optimized Structures of N-MethylSubstituted Cyclic Amines and Their N-Protonated lons

The imaginary frequency number is shown in parentheses. The natural population charges are shown in italic. 
Table S1. The Coordinates of Atoms in $\mathbf{1}$ Total Energy (au):-133.919399408(B3LYP/6-31G*)

\begin{tabular}{|c|c|c|c|}
\hline atom & $\mathrm{X}$ & $\mathrm{y}$ & $\mathrm{Z}$ \\
\hline $\mathrm{N}$ & 0.000000 & 0.000000 & 0.000000 \\
\hline $\mathrm{H}$ & -0.877449 & 0.000000 & -0.519987 \\
\hline $\mathrm{C}$ & 1.026910 & -0.742413 & -0.750940 \\
\hline $\mathrm{C}$ & 1.026910 & 0.742413 & -0.750940 \\
\hline $\mathrm{H}$ & 1.737588 & 1.287514 & -0.133791 \\
\hline $\mathrm{H}$ & 1.737588 & -1.287514 & -0.133791 \\
\hline $\mathrm{H}$ & 0.744441 & 1.254627 & -1.669681 \\
\hline $\mathrm{H}$ & 0.744441 & -1.254627 & -1.669681 \\
\hline
\end{tabular}

Table S2. The Coordinates of Atoms in 2 Total Energy (au):-173.237721346(B3LYP/6-31G*)

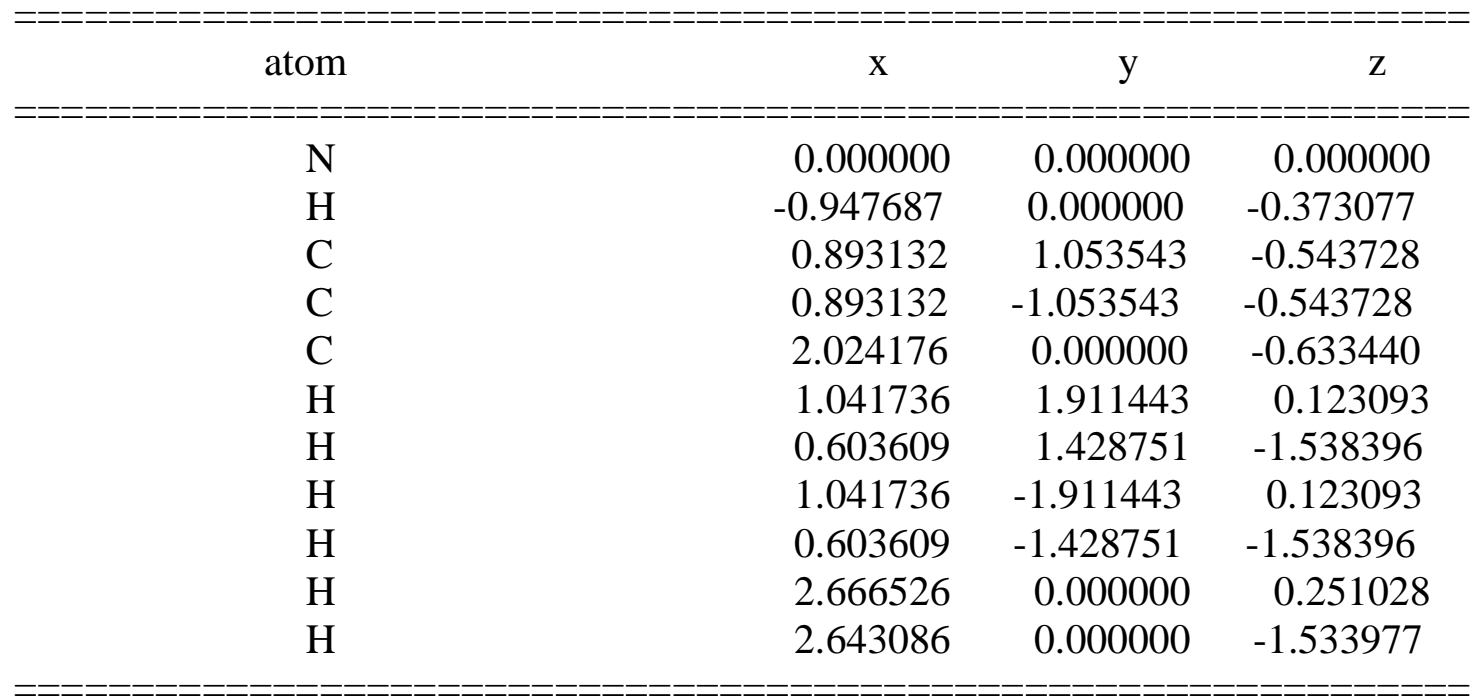

Table S3. The Coordinates of Atoms in $\mathbf{3}$ Total Energy (au):-212.581750298(B3LYP/6-31G*)

\begin{tabular}{|c|c|c|c|}
\hline atom & $\mathrm{X}$ & $\mathrm{y}$ & $\mathrm{Z}$ \\
\hline $\mathrm{N}$ & 0.000000 & 0.000000 & 0.000000 \\
\hline $\mathrm{H}$ & -0.960551 & 0.000000 & -0.337377 \\
\hline $\mathrm{C}$ & 0.750415 & 1.161668 & -0.485743 \\
\hline $\mathrm{C}$ & 0.750415 & -1.161668 & -0.485743 \\
\hline $\mathrm{C}$ & 2.212770 & 0.778622 & -0.173745 \\
\hline $\mathrm{C}$ & 2.212770 & -0.778622 & -0.173745 \\
\hline $\mathrm{H}$ & 0.426526 & 2.075068 & 0.025375 \\
\hline
\end{tabular}


Supporting Information

\begin{tabular}{|c|c|c|c|}
\hline $\mathrm{H}$ & 0.632206 & 1.326210 & -1.574317 \\
\hline $\mathrm{H}$ & 0.426526 & -2.075068 & 0.025375 \\
\hline $\mathrm{H}$ & 0.632206 & -1.326210 & -1.574317 \\
\hline $\mathrm{H}$ & 2.506327 & 1.161115 & 0.808406 \\
\hline $\mathrm{H}$ & 2.903947 & 1.199877 & -0.910269 \\
\hline $\mathrm{H}$ & 2.506327 & -1.161115 & 0.808406 \\
\hline $\mathrm{H}$ & 2.903947 & -1.199877 & -0.910269 \\
\hline
\end{tabular}

Table S4. The Coordinates of Atoms in 4 Total Energy (au):-251.904371463(B3LYP/6-31G*)

\begin{tabular}{|c|c|c|c|}
\hline atom & $\mathrm{x}$ & $\mathrm{y}$ & $\mathrm{Z}$ \\
\hline \multirow{2}{*}{\multicolumn{4}{|c|}{$==-==-==-==-$}} \\
\hline & 0.000000 & 0.000000 & 0.000000 \\
\hline $\mathrm{H}$ & -0.965825 & 0.000000 & -0.320513 \\
\hline $\mathrm{C}$ & 0.675416 & -1.214662 & -0.461216 \\
\hline $\mathrm{C}$ & 0.675416 & 1.214662 & -0.461216 \\
\hline $\mathrm{C}$ & 2.093437 & -1.265378 & 0.119293 \\
\hline $\mathrm{C}$ & 2.093437 & 1.265378 & 0.119293 \\
\hline $\mathrm{C}$ & 2.878969 & 0.000000 & -0.260161 \\
\hline $\mathrm{H}$ & 0.094620 & -2.082603 & -0.127153 \\
\hline $\mathrm{H}$ & 0.740326 & -1.269977 & -1.567743 \\
\hline $\mathrm{H}$ & 0.094620 & 2.082603 & -0.127153 \\
\hline $\mathrm{H}$ & 0.740326 & 1.269977 & -1.567743 \\
\hline $\mathrm{H}$ & 2.021002 & -1.341334 & 1.211995 \\
\hline $\mathrm{H}$ & 2.611113 & -2.163906 & -0.239868 \\
\hline $\mathrm{H}$ & 2.021002 & 1.341334 & 1.211995 \\
\hline $\mathrm{H}$ & 2.611113 & 2.163906 & -0.239868 \\
\hline $\mathrm{H}$ & 3.865847 & 0.000000 & 0.219532 \\
\hline $\mathrm{H}$ & 3.057765 & 0.000000 & -1.345887 \\
\hline
\end{tabular}

Table S5. The Coordinates of Atoms in 5 Total Energy (au):-173.231338793(B3LYP/6-31G*)

\begin{tabular}{|c|c|c|c|}
\hline atom & $\mathrm{X}$ & $\mathrm{y}$ & Z \\
\hline $\mathrm{N}$ & 0.000000 & 0.000000 & 0.000000 \\
\hline $\mathrm{C}$ & 1.296508 & 0.000000 & -0.664278 \\
\hline $\mathrm{C}$ & -1.064176 & 0.745664 & -0.665768 \\
\hline $\mathrm{C}$ & -1.064176 & -0.745664 & -0.665768 \\
\hline $\mathrm{H}$ & -1.733815 & -1.301895 & -0.012962 \\
\hline $\mathrm{H}$ & -0.831926 & -1.242129 & -1.609521 \\
\hline $\mathrm{H}$ & -1.733815 & 1.301895 & -0.012962 \\
\hline
\end{tabular}


Supporting Information

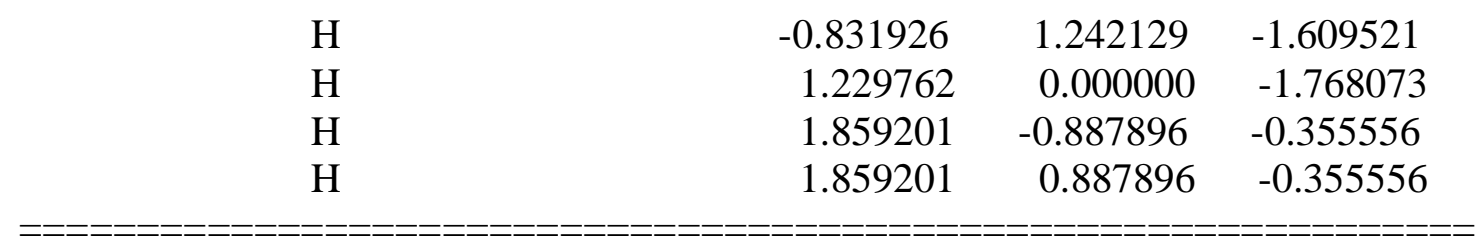

Table S6. The Coordinates of Atoms in 6 Total Energy (au):-212.552267848(B3LYP/6-31G*)

\begin{tabular}{|c|c|c|c|}
\hline atom & $\mathrm{X}$ & $\mathrm{y}$ & $\mathrm{Z}$ \\
\hline $\mathrm{N}$ & 0.000000 & 0.000000 & 0.000000 \\
\hline $\mathrm{C}$ & -1.365659 & 0.000000 & -0.476917 \\
\hline $\mathrm{C}$ & 0.918676 & 1.049612 & -0.487117 \\
\hline $\mathrm{C}$ & 0.918676 & -1.049612 & -0.487117 \\
\hline $\mathrm{C}$ & 2.055481 & 0.000000 & -0.503443 \\
\hline $\mathrm{H}$ & -1.442377 & 0.000000 & -1.583216 \\
\hline $\mathrm{H}$ & -1.889952 & -0.885849 & -0.099497 \\
\hline $\mathrm{H}$ & -1.889952 & 0.885849 & -0.099497 \\
\hline $\mathrm{H}$ & 1.016669 & 1.921079 & 0.171834 \\
\hline $\mathrm{H}$ & 0.672991 & 1.400687 & -1.505789 \\
\hline $\mathrm{H}$ & 1.016669 & -1.921079 & 0.171834 \\
\hline $\mathrm{H}$ & 0.672991 & -1.400687 & -1.505789 \\
\hline $\mathrm{H}$ & 2.641764 & 0.000000 & 0.418883 \\
\hline $\mathrm{H}$ & 2.726120 & 0.000000 & -1.365528 \\
\hline
\end{tabular}

Table S7. The Coordinates of Atoms in 7 Total Energy (au):-251.895004235(B3LYP/6-31G*)

\begin{tabular}{|c|c|c|c|}
\hline atom & $\mathrm{x}$ & $\mathrm{y}$ & $\mathrm{Z}$ \\
\hline $\mathrm{N}$ & 0.000000 & 0.000000 & 0.000000 \\
\hline $\mathrm{C}$ & -1.380470 & 0.000000 & -0.440954 \\
\hline $\mathrm{C}$ & 0.773449 & 1.157522 & -0.444685 \\
\hline $\mathrm{C}$ & 0.773449 & -1.157522 & -0.444685 \\
\hline $\mathrm{C}$ & 2.228094 & 0.778661 & -0.105796 \\
\hline $\mathrm{C}$ & 2.228094 & -0.778661 & -0.105796 \\
\hline $\mathrm{H}$ & -1.482933 & 0.000000 & -1.545523 \\
\hline $\mathrm{H}$ & -1.895472 & -0.886213 & -0.053201 \\
\hline $\mathrm{H}$ & -1.895472 & 0.886213 & -0.053201 \\
\hline $\mathrm{H}$ & 0.430298 & 2.067977 & 0.060449 \\
\hline $\mathrm{H}$ & 0.665036 & 1.323607 & -1.537074 \\
\hline $\mathrm{H}$ & 0.430298 & -2.067977 & 0.060449 \\
\hline $\mathrm{H}$ & 0.665036 & -1.323607 & -1.537074 \\
\hline
\end{tabular}


Supporting Information

\begin{tabular}{lrrr}
$\mathrm{H}$ & 2.506000 & 1.162082 & 0.880638 \\
$\mathrm{H}$ & 2.931743 & 1.199448 & -0.830634 \\
$\mathrm{H}$ & 2.506000 & -1.162082 & 0.880638 \\
$\mathrm{H}$ & 2.931743 & -1.199448 & -0.830634 \\
\hline
\end{tabular}

Table S8. The Coordinates of Atoms in $\mathbf{8}$ Total Energy (au):-291.216335388(B3LYP/6-31G*)

\begin{tabular}{|c|c|c|c|}
\hline atom & $\mathrm{x}$ & $\mathrm{y}$ & $\mathrm{Z}$ \\
\hline & & & \\
\hline $\mathrm{N}$ & 0.000000 & 0.000000 & 0.000000 \\
\hline $\mathrm{C}$ & -1.388363 & 0.000000 & -0.431906 \\
\hline $\mathrm{C}$ & 0.699667 & -1.209078 & -0.434570 \\
\hline $\mathrm{C}$ & 0.699667 & 1.209078 & -0.434570 \\
\hline $\mathrm{C}$ & 2.122066 & -1.258561 & 0.131767 \\
\hline $\mathrm{C}$ & 2.122066 & 1.258561 & 0.131767 \\
\hline $\mathrm{C}$ & 2.909733 & 0.000000 & -0.257718 \\
\hline $\mathrm{H}$ & 0.742274 & -1.272044 & -1.543779 \\
\hline $\mathrm{H}$ & 0.126136 & -2.078339 & -0.089833 \\
\hline $\mathrm{H}$ & 0.742274 & 1.272044 & -1.543779 \\
\hline $\mathrm{H}$ & 0.126136 & 2.078339 & -0.089833 \\
\hline $\mathrm{H}$ & 2.628930 & -2.162429 & -0.229172 \\
\hline $\mathrm{H}$ & 2.062129 & -1.331901 & 1.225500 \\
\hline $\mathrm{H}$ & 2.628930 & 2.162429 & -0.229172 \\
\hline $\mathrm{H}$ & 2.062129 & 1.331901 & 1.225500 \\
\hline $\mathrm{H}$ & 3.899838 & 0.000000 & 0.214969 \\
\hline $\mathrm{H}$ & 3.078457 & 0.000000 & -1.344879 \\
\hline $\mathrm{H}$ & -1.505356 & 0.000000 & -1.534691 \\
\hline $\mathrm{H}$ & -1.898290 & -0.887098 & -0.039413 \\
\hline $\mathrm{H}$ & -1.898290 & 0.887098 & -0.039413 \\
\hline
\end{tabular}

Table S9. The Coordinates of Atoms in 9 Total Energy (au):-364.974618290(B3LYP/6-31G*)

\begin{tabular}{|c|c|c|c|}
\hline atom & $\mathrm{X}$ & $\mathrm{y}$ & Z \\
\hline $\mathrm{N}$ & 0.000000 & 0.000000 & 0.000000 \\
\hline $\mathrm{C}$ & -1.285103 & 0.000000 & -0.585130 \\
\hline $\mathrm{C}$ & 1.094462 & -0.747442 & -0.603449 \\
\hline $\mathrm{C}$ & 1.094462 & 0.747442 & -0.603449 \\
\hline $\mathrm{H}$ & 1.727925 & 1.301113 & 0.086260 \\
\hline $\mathrm{H}$ & 0.905218 & 1.242900 & -1.555779 \\
\hline $\mathrm{H}$ & 0.905218 & -1.242900 & -1.555779 \\
\hline
\end{tabular}


Supporting Information

$\begin{array}{crrr}\text { H } & 1.727925 & -1.301113 & 0.086260 \\ \mathrm{C} & -1.947932 & 1.209846 & -0.841261 \\ \mathrm{C} & -1.947932 & -1.209846 & -0.841261 \\ \mathrm{C} & -3.246397 & 1.204757 & -1.348365 \\ \mathrm{C} & -3.246397 & -1.204757 & -1.348365 \\ \mathrm{C} & -3.903905 & 0.000000 & -1.606597 \\ \mathrm{H} & -1.440691 & 2.148238 & -0.634073 \\ \mathrm{H} & -1.440691 & -2.148238 & -0.634073 \\ \mathrm{H} & -3.745881 & 2.150333 & -1.544025 \\ \mathrm{H} & -3.745881 & -2.150333 & -1.544025 \\ \mathrm{H} & -4.915086 & 0.000000 & -2.003447\end{array}$

Table S10. The Coordinates of Atoms of $\mathbf{1 0}$ Total Energy (au):-404.299613915(B3LYP/6-31G*)

\begin{tabular}{|c|c|c|c|}
\hline atom & $\mathrm{X}$ & $\mathrm{y}$ & $\mathrm{Z}$ \\
\hline \multicolumn{4}{|c|}{ 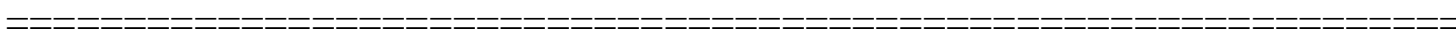 } \\
\hline $\mathrm{N}$ & 0.000000 & 0.000000 & 0.000000 \\
\hline $\mathrm{C}$ & -1.352291 & 0.000000 & -0.306141 \\
\hline $\mathrm{C}$ & 0.955629 & 1.069000 & -0.324610 \\
\hline $\mathrm{C}$ & 0.955629 & -1.069000 & -0.324610 \\
\hline $\mathrm{C}$ & 2.078623 & 0.000000 & -0.370634 \\
\hline $\mathrm{H}$ & 0.759343 & 1.546631 & -1.297254 \\
\hline $\mathrm{H}$ & 1.044567 & 1.851571 & 0.438852 \\
\hline $\mathrm{H}$ & 0.759343 & -1.546631 & -1.297254 \\
\hline $\mathrm{H}$ & 1.044567 & -1.851571 & 0.438852 \\
\hline $\mathrm{H}$ & 2.704187 & 0.000000 & -1.265393 \\
\hline $\mathrm{H}$ & 2.715863 & 0.000000 & 0.516735 \\
\hline $\mathrm{C}$ & -2.056134 & 1.211431 & -0.450251 \\
\hline $\mathrm{C}$ & -2.056134 & -1.211431 & -0.450251 \\
\hline $\mathrm{C}$ & -3.421243 & 1.202917 & -0.727191 \\
\hline $\mathrm{C}$ & -3.421243 & -1.202917 & -0.727191 \\
\hline $\mathrm{C}$ & -4.116611 & 0.000000 & -0.869382 \\
\hline $\mathrm{H}$ & -1.530508 & 2.155420 & -0.336451 \\
\hline $\mathrm{H}$ & -1.530508 & -2.155420 & -0.336451 \\
\hline $\mathrm{H}$ & -3.945324 & 2.149620 & -0.834578 \\
\hline $\mathrm{H}$ & -3.945324 & -2.149620 & -0.834578 \\
\hline $\mathrm{H}$ & -5.180773 & 0.000000 & -1.086378 \\
\hline
\end{tabular}

Table S11. The Coordinates of Atoms of 11e Total Energy (au):-443.638315686(B3LYP/6-31G*)

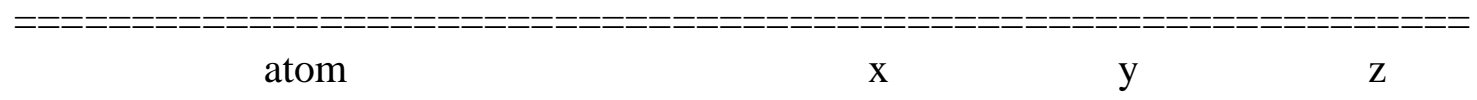


Supporting Information

\begin{tabular}{|c|c|c|c|}
\hline $\mathrm{N}$ & 0.000000 & 0.000000 & 0.000000 \\
\hline $\mathrm{C}$ & -1.371798 & 0.000000 & -0.245173 \\
\hline $\mathrm{C}$ & 0.802708 & -1.193746 & -0.257100 \\
\hline $\mathrm{C}$ & 0.802708 & 1.193746 & -0.257100 \\
\hline $\mathrm{C}$ & 2.248474 & -0.775892 & 0.075775 \\
\hline $\mathrm{C}$ & 2.248474 & 0.775892 & 0.075775 \\
\hline $\mathrm{H}$ & 0.467168 & -2.025100 & 0.371470 \\
\hline $\mathrm{H}$ & 0.710537 & -1.520800 & -1.308040 \\
\hline $\mathrm{H}$ & 0.467168 & 2.025100 & 0.371470 \\
\hline $\mathrm{H}$ & 0.710537 & 1.520800 & -1.308040 \\
\hline $\mathrm{H}$ & 2.540880 & -1.158023 & 1.058341 \\
\hline $\mathrm{H}$ & 2.952018 & -1.190685 & -0.652146 \\
\hline $\mathrm{H}$ & 2.540880 & 1.158023 & 1.058341 \\
\hline $\mathrm{H}$ & 2.952018 & 1.190685 & -0.652146 \\
\hline $\mathrm{C}$ & -2.094238 & -1.207820 & -0.362591 \\
\hline $\mathrm{C}$ & -2.094238 & 1.207820 & -0.362591 \\
\hline $\mathrm{C}$ & -3.472402 & -1.199302 & -0.564168 \\
\hline $\mathrm{C}$ & -3.472402 & 1.199302 & -0.564168 \\
\hline $\mathrm{C}$ & -4.178584 & 0.000000 & -0.665850 \\
\hline $\mathrm{H}$ & -1.579179 & -2.159263 & -0.293144 \\
\hline $\mathrm{H}$ & -1.579179 & 2.159263 & -0.293144 \\
\hline $\mathrm{H}$ & -3.996672 & -2.148407 & -0.647373 \\
\hline $\mathrm{H}$ & -3.996672 & 2.148407 & -0.647373 \\
\hline $\mathrm{H}$ & -5.252701 & 0.000000 & -0.826390 \\
\hline
\end{tabular}

Table S12. The Coordinates of Atoms of 11 hc Total Energy (au):-443.641926091(B3LYP/6-31G*)

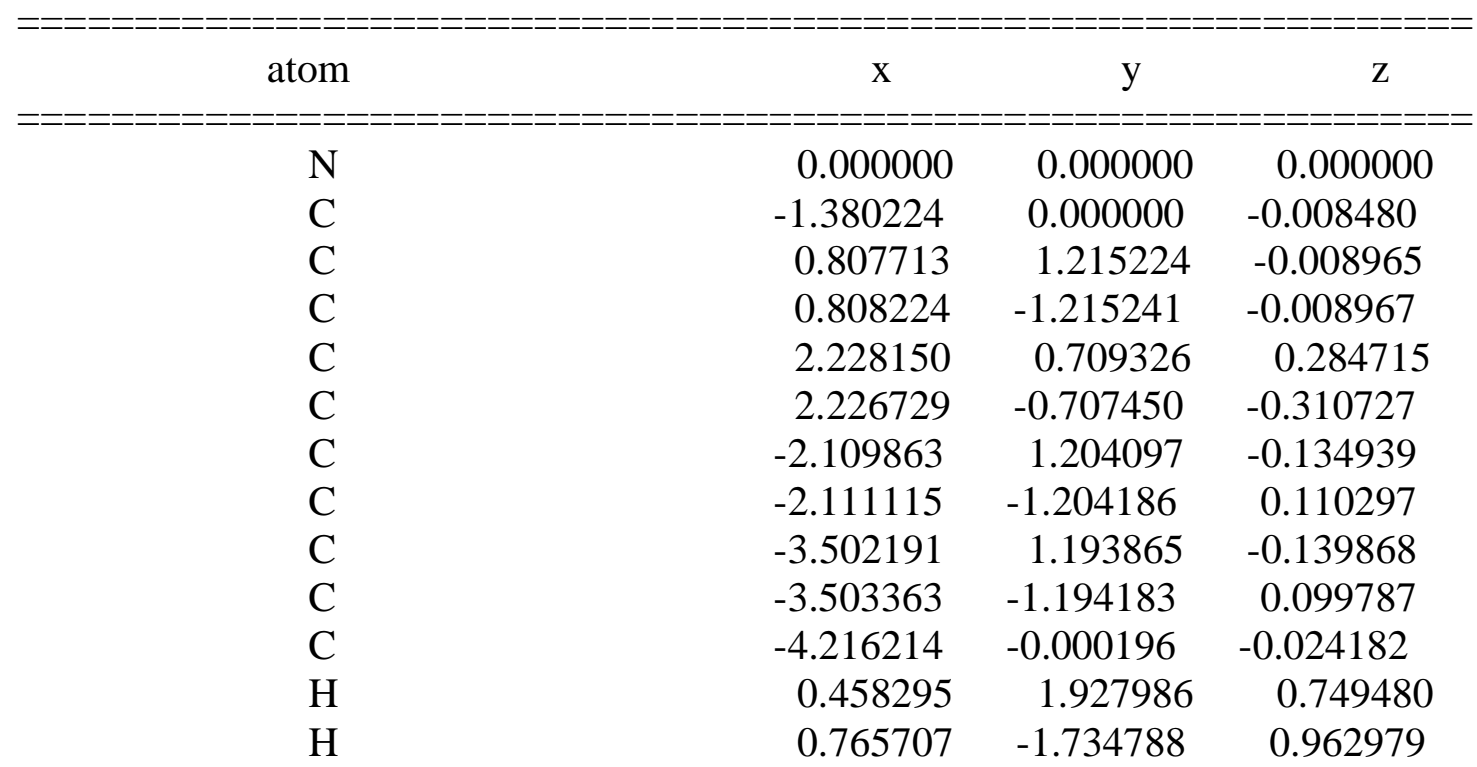


Supporting Information

\begin{tabular}{crrr} 
H & 0.757114 & 1.726776 & -0.984958 \\
H & 0.452421 & -1.921842 & -0.770305 \\
H & 2.390014 & 0.662908 & 1.368279 \\
H & 3.001509 & -1.355351 & 0.110178 \\
H & 2.999632 & 1.357451 & -0.141856 \\
H & 2.381957 & -0.659931 & -1.395240 \\
H & -1.584837 & 2.147447 & -0.240659 \\
H & -1.587046 & -2.147314 & 0.222722 \\
H & -4.033637 & 2.137374 & -0.240750 \\
H & -4.035790 & -2.137724 & 0.195008 \\
I & -5.302179 & -0.000266 & -0.030254 \\
\hline
\end{tabular}

Table S13 The Coordinates of Atoms of 12tw Total Energy (au):-482.955715210(B3LYP/6-31G*)

\begin{tabular}{|c|c|c|c|}
\hline \multirow{2}{*}{ atom } & $\mathrm{X}$ & $\mathrm{y}$ & $\mathrm{Z}$ \\
\hline & $========$ & $===$ & $=====$ \\
\hline $\mathrm{N}$ & 0.000000 & 0.000000 & 0.000000 \\
\hline $\mathrm{C}$ & -1.382383 & 0.000000 & -0.301814 \\
\hline $\mathrm{C}$ & 0.764736 & -1.205954 & -0.311770 \\
\hline $\mathrm{C}$ & 0.742221 & 1.230337 & -0.313712 \\
\hline $\mathrm{C}$ & 2.095246 & -1.220198 & 0.451528 \\
\hline $\mathrm{C}$ & 2.063978 & 1.292494 & 0.456504 \\
\hline $\mathrm{C}$ & 2.914030 & 0.046766 & 0.177098 \\
\hline $\mathrm{C}$ & -2.210351 & 1.005491 & 0.238510 \\
\hline $\mathrm{C}$ & -3.577620 & 1.021379 & -0.013918 \\
\hline $\mathrm{C}$ & -4.170189 & 0.028313 & -0.799919 \\
\hline $\mathrm{C}$ & -3.364829 & -0.976696 & -1.330265 \\
\hline $\mathrm{C}$ & -1.987912 & -0.991333 & -1.094835 \\
\hline $\mathrm{H}$ & 0.964180 & -1.291503 & -1.398685 \\
\hline $\mathrm{H}$ & 0.168803 & -2.076477 & -0.021980 \\
\hline $\mathrm{H}$ & 0.949343 & 1.283426 & -1.400702 \\
\hline $\mathrm{H}$ & 0.121201 & 2.093655 & -0.068066 \\
\hline $\mathrm{H}$ & 2.659937 & -2.117611 & 0.169020 \\
\hline $\mathrm{H}$ & 1.880906 & -1.294495 & 1.525581 \\
\hline $\mathrm{H}$ & 2.604341 & 2.204788 & 0.173809 \\
\hline $\mathrm{H}$ & 1.845797 & 1.359413 & 1.530520 \\
\hline $\mathrm{H}$ & 3.233062 & 0.054317 & -0.875724 \\
\hline $\mathrm{H}$ & 3.827797 & 0.055094 & 0.783792 \\
\hline $\mathrm{H}$ & -1.777177 & 1.760969 & 0.887363 \\
\hline $\mathrm{H}$ & -4.188873 & 1.807642 & 0.422385 \\
\hline $\mathrm{H}$ & -5.239836 & 0.038180 & -0.988843 \\
\hline $\mathrm{H}$ & -3.803029 & -1.757380 & -1.947454 \\
\hline
\end{tabular}


$\mathrm{H} \quad-1.387146 \quad-1.772780 \quad-1.546580$

Table S14. The Coordinates of Atoms of 12p

Total Energy (au):-482.953521685(B3LYP/6-31G*)

\begin{tabular}{|c|c|c|c|}
\hline atom & $\mathrm{X}$ & $\mathrm{y}$ & $\mathrm{Z}$ \\
\hline $\mathrm{N}$ & 0.000000 & 0.000000 & 0.000000 \\
\hline $\mathrm{C}$ & -1.384815 & 0.000000 & -0.276282 \\
\hline $\mathrm{C}$ & 0.742760 & -1.233471 & -0.287260 \\
\hline $\mathrm{C}$ & 0.742760 & 1.233471 & -0.287260 \\
\hline $\mathrm{C}$ & 2.151369 & -1.239931 & 0.314922 \\
\hline $\mathrm{C}$ & 2.151369 & 1.239931 & 0.314922 \\
\hline $\mathrm{C}$ & 2.947740 & 0.000000 & -0.091403 \\
\hline $\mathrm{C}$ & -2.118946 & -1.201790 & -0.406923 \\
\hline $\mathrm{C}$ & -2.118946 & 1.201790 & -0.406923 \\
\hline $\mathrm{C}$ & -3.495673 & -1.194786 & -0.620147 \\
\hline $\mathrm{C}$ & -3.495673 & 1.194786 & -0.620147 \\
\hline $\mathrm{C}$ & -4.205344 & 0.000000 & -0.727089 \\
\hline $\mathrm{H}$ & 0.803739 & -1.406945 & -1.378465 \\
\hline $\mathrm{H}$ & 0.199271 & -2.077078 & 0.140306 \\
\hline $\mathrm{H}$ & 0.803739 & 1.406945 & -1.378465 \\
\hline $\mathrm{H}$ & 0.199271 & 2.077078 & 0.140306 \\
\hline $\mathrm{H}$ & 2.656675 & -2.160418 & -0.002748 \\
\hline $\mathrm{H}$ & 2.070942 & -1.276909 & 1.409634 \\
\hline $\mathrm{H}$ & 2.656675 & 2.160418 & -0.002748 \\
\hline $\mathrm{H}$ & 2.070942 & 1.276909 & 1.409634 \\
\hline $\mathrm{H}$ & 3.109111 & 0.000000 & -1.179448 \\
\hline $\mathrm{H}$ & 3.938648 & 0.000000 & 0.378641 \\
\hline $\mathrm{H}$ & -1.626315 & -2.163204 & -0.344418 \\
\hline $\mathrm{H}$ & -1.626315 & 2.163204 & -0.344418 \\
\hline $\mathrm{H}$ & -4.013499 & -2.146860 & -0.709563 \\
\hline $\mathrm{H}$ & -4.013499 & 2.146860 & -0.709563 \\
\hline $\mathrm{H}$ & -5.278048 & 0.000000 & -0.896552 \\
\hline
\end{tabular}

Table S15. The Coordinates of Atoms of $\mathbf{1 2 v}$

Total Energy (au):-482.951830202(B3LYP/6-31G*)

\begin{tabular}{|c|c|c|c|}
\hline atom & $\mathrm{X}$ & $\mathrm{y}$ & $\mathrm{Z}$ \\
\hline $\mathrm{N}$ & 0.000000 & 0.000000 & 0.000000 \\
\hline $\mathrm{C}$ & -1.387577 & 0.000000 & -0.385187 \\
\hline $\mathrm{C}$ & 0.720898 & -1.218674 & -0.393058 \\
\hline
\end{tabular}


Supporting Information

$\begin{array}{lrrr}\mathrm{C} & 0.720898 & 1.218674 & -0.393058 \\ \mathrm{C} & 2.100777 & -1.260004 & 0.272447 \\ \mathrm{C} & 2.100777 & 1.260004 & 0.272447 \\ \mathrm{C} & 2.909494 & 0.000000 & -0.065632 \\ \mathrm{C} & -2.359037 & 0.000000 & 0.621576 \\ \mathrm{C} & -3.717172 & 0.000000 & 0.298477 \\ \mathrm{C} & -4.118818 & 0.000000 & -1.038516 \\ \mathrm{C} & -3.155598 & 0.000000 & -2.049917 \\ \mathrm{C} & -1.798178 & 0.000000 & -1.726343 \\ \mathrm{H} & 0.845922 & -1.286417 & -1.492986 \\ \mathrm{H} & 0.120983 & -2.081644 & -0.084996 \\ \mathrm{H} & 0.845922 & 1.286417 & -1.492986 \\ \mathrm{H} & 0.120983 & 2.081644 & -0.084996 \\ \mathrm{H} & 2.634740 & -2.163176 & -0.049349 \\ \mathrm{H} & 1.963556 & -1.329083 & 1.359386 \\ \mathrm{H} & 2.634740 & 2.163176 & -0.049349 \\ \mathrm{H} & 1.963556 & 1.329083 & 1.359386 \\ \mathrm{H} & 3.147994 & 0.000000 & -1.139827 \\ \mathrm{H} & 3.867379 & 0.000000 & 0.469119 \\ \mathrm{H} & -2.027461 & 0.000000 & 1.655855 \\ \mathrm{H} & -4.461058 & 0.000000 & 1.091141 \\ \mathrm{H} & -5.175701 & 0.000000 & -1.291892 \\ \mathrm{H} & -3.462096 & 0.000000 & -3.093076 \\ \mathrm{H}=======================================\end{array}$

Table S16. The Coordinates of Atoms of N-Protonated 1 Total Energy (au):-134.282777046(B3LYP/6-31G*)

\begin{tabular}{|c|c|c|c|}
\hline atom & $\mathrm{x}$ & $\mathrm{y}$ & $\mathrm{Z}$ \\
\hline $\mathrm{N}$ & -0.000045 & 0.824733 & 0.000000 \\
\hline $\mathrm{H}$ & 0.852436 & 1.385866 & 0.000000 \\
\hline $\mathrm{H}$ & -0.852649 & 1.385880 & 0.000000 \\
\hline $\mathrm{C}$ & -0.000045 & -0.484797 & 0.738911 \\
\hline $\mathrm{C}$ & -0.000045 & -0.484797 & -0.738911 \\
\hline $\mathrm{H}$ & 0.921791 & -0.681718 & -1.276241 \\
\hline $\mathrm{H}$ & 0.921791 & -0.681718 & 1.276241 \\
\hline $\mathrm{H}$ & -0.921255 & -0.681938 & -1.277210 \\
\hline $\mathrm{H}$ & -0.921255 & -0.681938 & 1.277210 \\
\hline
\end{tabular}

Table S17. The Coordinates of Atoms of N-Protonated 2 Total Energy (au):-173.614263818(B3LYP/6-31G*) 
Supporting Information

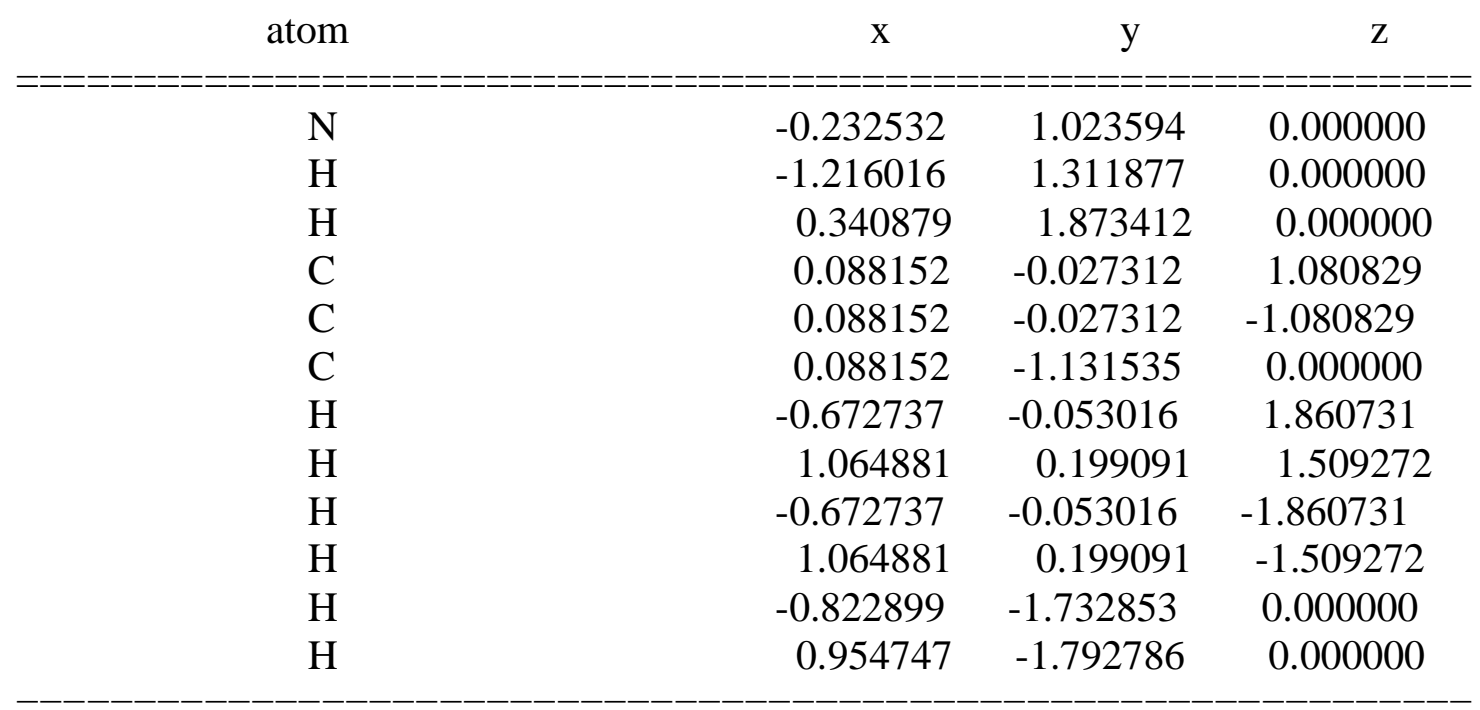

Table S18. The Coordinates of Atoms of N-Protonated 3 Total Energy (au):-212.961488503(B3LYP/6-31G*)

\begin{tabular}{|c|c|c|c|}
\hline \multirow{2}{*}{$=========+$ atom } & $\mathrm{x}$ & $\mathrm{y}$ & $\mathrm{z}$ \\
\hline & & & \\
\hline $\mathrm{N}$ & 0.453862 & -1.135915 & 0.000000 \\
\hline $\mathrm{H}$ & 1.476513 & -1.048376 & 0.000000 \\
\hline $\mathrm{H}$ & 0.243041 & -2.139710 & 0.000000 \\
\hline $\mathrm{C}$ & -0.128361 & -0.413991 & 1.208551 \\
\hline $\mathrm{C}$ & -0.128361 & -0.413991 & -1.208551 \\
\hline $\mathrm{C}$ & -0.128361 & 1.061842 & 0.779916 \\
\hline $\mathrm{C}$ & -0.128361 & 1.061842 & -0.779916 \\
\hline $\mathrm{H}$ & 0.473635 & -0.646614 & 2.088321 \\
\hline $\mathrm{H}$ & -1.135409 & -0.814971 & 1.343555 \\
\hline $\mathrm{H}$ & 0.473635 & -0.646614 & -2.088321 \\
\hline $\mathrm{H}$ & -1.135409 & -0.814971 & -1.343555 \\
\hline $\mathrm{H}$ & 0.755975 & 1.574929 & 1.166439 \\
\hline $\mathrm{H}$ & -1.002159 & 1.569293 & 1.193780 \\
\hline $\mathrm{H}$ & 0.755975 & 1.574929 & -1.166439 \\
\hline $\mathrm{H}$ & -1.002159 & 1.569293 & -1.193780 \\
\hline
\end{tabular}

Table S19 The Coordinates of Atoms of N-Protonated 4 Total Energy (au):-252.285940225(B3LYP/6-31G*)

\begin{tabular}{|c|c|c|c|}
\hline atom & $\mathrm{X}$ & $\mathrm{y}$ & $\mathrm{Z}$ \\
\hline$====$ $=$ = = = =ニ= = & $=======$ & $=======$ & $=======$ \\
\hline $\mathrm{N}$ & -0.645560 & -1.259783 & 0.000000 \\
\hline $\mathrm{H}$ & -1.632515 & -0.974576 & 0.000000 \\
\hline
\end{tabular}

S13 
Supporting Information

\begin{tabular}{cccc}
$\mathrm{H}$ & -0.643964 & -2.285926 & 0.000000 \\
$\mathrm{C}$ & 0.015307 & -0.741193 & 1.272844 \\
$\mathrm{C}$ & 0.015307 & -0.741193 & -1.272844 \\
$\mathrm{C}$ & 0.015307 & 0.785057 & 1.268247 \\
$\mathrm{C}$ & 0.015307 & 0.785057 & -1.268247 \\
$\mathrm{C}$ & 0.674480 & 1.350317 & 0.000000 \\
$\mathrm{H}$ & -0.537817 & -1.167079 & 2.113543 \\
$\mathrm{H}$ & 1.028494 & -1.153095 & 1.269666 \\
$\mathrm{H}$ & -0.537817 & -1.167079 & -2.113543 \\
$\mathrm{H}$ & 1.028494 & -1.153095 & -1.269666 \\
$\mathrm{H}$ & -1.016402 & 1.151655 & 1.361331 \\
$\mathrm{H}$ & 0.543119 & 1.119813 & 2.167269 \\
$\mathrm{H}$ & -1.016402 & 1.151655 & -1.361331 \\
$\mathrm{H}$ & 0.543119 & 1.119813 & -2.167269 \\
$\mathrm{H}$ & 0.601406 & 2.441665 & 0.000000 \\
$\mathrm{H}$ & 1.744963 & 1.106465 & 0.000000 \\
\hline$=============================================$
\end{tabular}

Table S20. The Coordinates of Atoms of N-Protonated 5 Total Energy (au):-173.603502573(B3LYP/6-31G*)

\begin{tabular}{|c|c|c|c|}
\hline atom & $\mathrm{x}$ & $\mathrm{y}$ & $\mathrm{Z}$ \\
\hline $\mathrm{C}$ & -1486185 & 0.415932 & 0.000000 \\
\hline $\mathrm{N}$ & 0.000000 & 0.493107 & 0.000000 \\
\hline $\mathrm{C}$ & 0.846294 & -0.497100 & 0.740474 \\
\hline $\mathrm{C}$ & 0.846294 & -0.497100 & -0.740474 \\
\hline $\mathrm{H}$ & 0.359390 & 1.448116 & 0.000000 \\
\hline $\mathrm{H}$ & 1.681338 & -0.061886 & 1.279883 \\
\hline $\mathrm{H}$ & 0.271720 & -1.246657 & 1.274983 \\
\hline $\mathrm{H}$ & 1.681338 & -0.061886 & -1.279883 \\
\hline $\mathrm{H}$ & 0.271720 & -1.246657 & -1.274983 \\
\hline $\mathrm{H}$ & -1.782519 & -0.634059 & 0.000000 \\
\hline $\mathrm{H}$ & -1.860705 & 0.910444 & 0.898053 \\
\hline $\mathrm{H}$ & -1.860705 & 0.910444 & -0.898053 \\
\hline
\end{tabular}

Table S21. The Coordinates of Atoms of N-Protonated 6 Total Energy (au):-212.933802707(B3LYP/6-31G*)

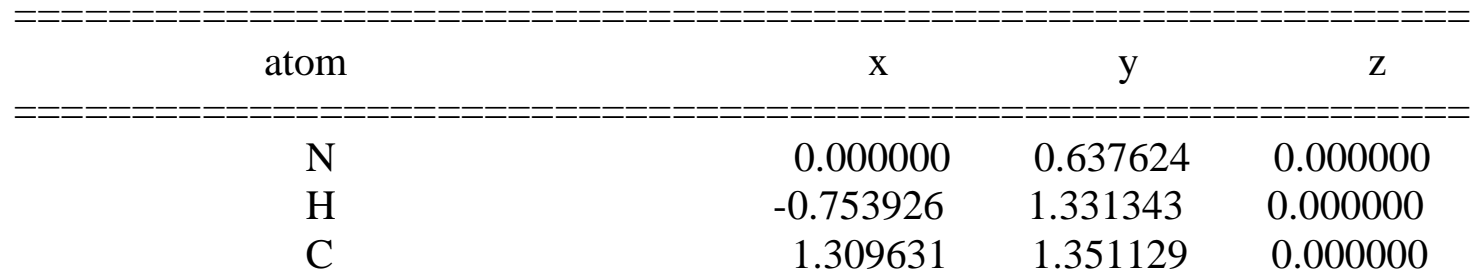


Supporting Information

\begin{tabular}{lccc}
$\mathrm{C}$ & -0.284085 & -0.425576 & 1.074339 \\
$\mathrm{C}$ & -0.284085 & -0.425576 & -1.074339 \\
$\mathrm{C}$ & -0.915452 & -1.336659 & 0.000000 \\
$\mathrm{H}$ & 2.110820 & 0.610507 & 0.000000 \\
$\mathrm{H}$ & 1.374122 & 1.973248 & -0.895187 \\
$\mathrm{H}$ & 1.374122 & 1.973248 & 0.895187 \\
$\mathrm{H}$ & -0.906308 & -0.042976 & 1.884187 \\
$\mathrm{H}$ & 0.670575 & -0.781435 & 1.465306 \\
$\mathrm{H}$ & -0.906308 & -0.042976 & -1.884187 \\
$\mathrm{H}$ & 0.670575 & -0.781435 & -1.465306 \\
$\mathrm{H}$ & -2.006895 & -1.308167 & 0.000000 \\
$\mathrm{H}$ & -0.582823 & -2.374629 & 0.000000 \\
\hline$=========================================$
\end{tabular}

Table S22. The Coordinates of Atoms of N-Protonated 7 Total Energy (au):-252.278823539(B3LYP/6-31G*)

\begin{tabular}{|c|c|c|c|}
\hline atom & $\mathrm{X}$ & $\mathrm{y}$ & $\mathrm{Z}$ \\
\hline $\mathrm{N}$ & 0.000000 & 0.801225 & 0.000000 \\
\hline $\mathrm{H}$ & -0.955747 & 1.174948 & 0.000000 \\
\hline $\mathrm{C}$ & 0.945916 & 1.963714 & 0.000000 \\
\hline $\mathrm{C}$ & 0.122546 & -0.127770 & 1.197526 \\
\hline $\mathrm{C}$ & 0.122546 & -0.127770 & -1.197526 \\
\hline $\mathrm{C}$ & -0.654918 & -1.384382 & 0.780013 \\
\hline $\mathrm{C}$ & -0.654918 & -1.384382 & -0.780013 \\
\hline $\mathrm{H}$ & 1.966780 & 1.578692 & 0.000000 \\
\hline $\mathrm{H}$ & 0.770971 & 2.563668 & -0.894533 \\
\hline $\mathrm{H}$ & 0.770971 & 2.563668 & 0.894533 \\
\hline $\mathrm{H}$ & -0.256165 & 0.383807 & 2.084427 \\
\hline $\mathrm{H}$ & 1.191941 & -0.316471 & 1.323060 \\
\hline $\mathrm{H}$ & -0.256165 & 0.383807 & -2.084427 \\
\hline $\mathrm{H}$ & 1.191941 & -0.316471 & -1.323060 \\
\hline $\mathrm{H}$ & -1.676603 & -1.354965 & 1.167772 \\
\hline $\mathrm{H}$ & -0.179177 & -2.275375 & 1.194986 \\
\hline $\mathrm{H}$ & -1.676603 & -1.354965 & -1.167772 \\
\hline $\mathrm{H}$ & -0.179177 & -2.275375 & -1.194986 \\
\hline
\end{tabular}

Table S23 The Coordinates of Atoms in N-Protonated 8 Total Energy (au):-291.602258992(B3LYP/6-31G*)

\begin{tabular}{|c|c|c|c|}
\hline atom & $\mathrm{x}$ & $\mathrm{y}$ & $\mathrm{Z}$ \\
\hline $\mathrm{N}$ & 0.000000 & 1.021036 & 0.000000 \\
\hline
\end{tabular}


Supporting Information

\begin{tabular}{crrr}
$\mathrm{H}$ & 1.005576 & 1.229294 & 0.000000 \\
$\mathrm{C}$ & -0.732937 & 2.331950 & 0.000000 \\
$\mathrm{C}$ & -0.278797 & 0.207677 & 1.256707 \\
$\mathrm{C}$ & -0.278797 & 0.207677 & -1.256707 \\
$\mathrm{C}$ & 0.541832 & -1.079798 & 1.263278 \\
$\mathrm{C}$ & 0.541832 & -1.079798 & -1.263278 \\
$\mathrm{C}$ & 0.297174 & -1.917956 & 0.000000 \\
$\mathrm{H}$ & -1.354880 & 0.008559 & 1.247839 \\
$\mathrm{H}$ & -0.049818 & 0.854131 & 2.107872 \\
$\mathrm{H}$ & -1.354880 & 0.008559 & -1.247839 \\
$\mathrm{H}$ & -0.049818 & 0.854131 & -2.107872 \\
$\mathrm{H}$ & 0.273630 & -1.639282 & 2.165685 \\
$\mathrm{H}$ & 1.608688 & -0.833957 & 1.359404 \\
$\mathrm{H}$ & 0.273630 & -1.639282 & -2.165685 \\
$\mathrm{H}$ & 1.608688 & -0.833957 & -1.359404 \\
$\mathrm{H}$ & 0.952569 & -2.793777 & 0.000000 \\
$\mathrm{H}$ & -0.733946 & -2.295324 & 0.000000 \\
$\mathrm{H}$ & -1.805182 & 2.129966 & 0.000000 \\
$\mathrm{H}$ & -0.458053 & 2.892587 & 0.894889 \\
$\mathrm{H}$ & -0.458053 & 2.892587 & -0.894889 \\
\hline$============================================$
\end{tabular}

Table S24. The Coordinates of Atoms of N-Protonated 9 Total Energy (au):-365.344352597(B3LYP/6-31G*)

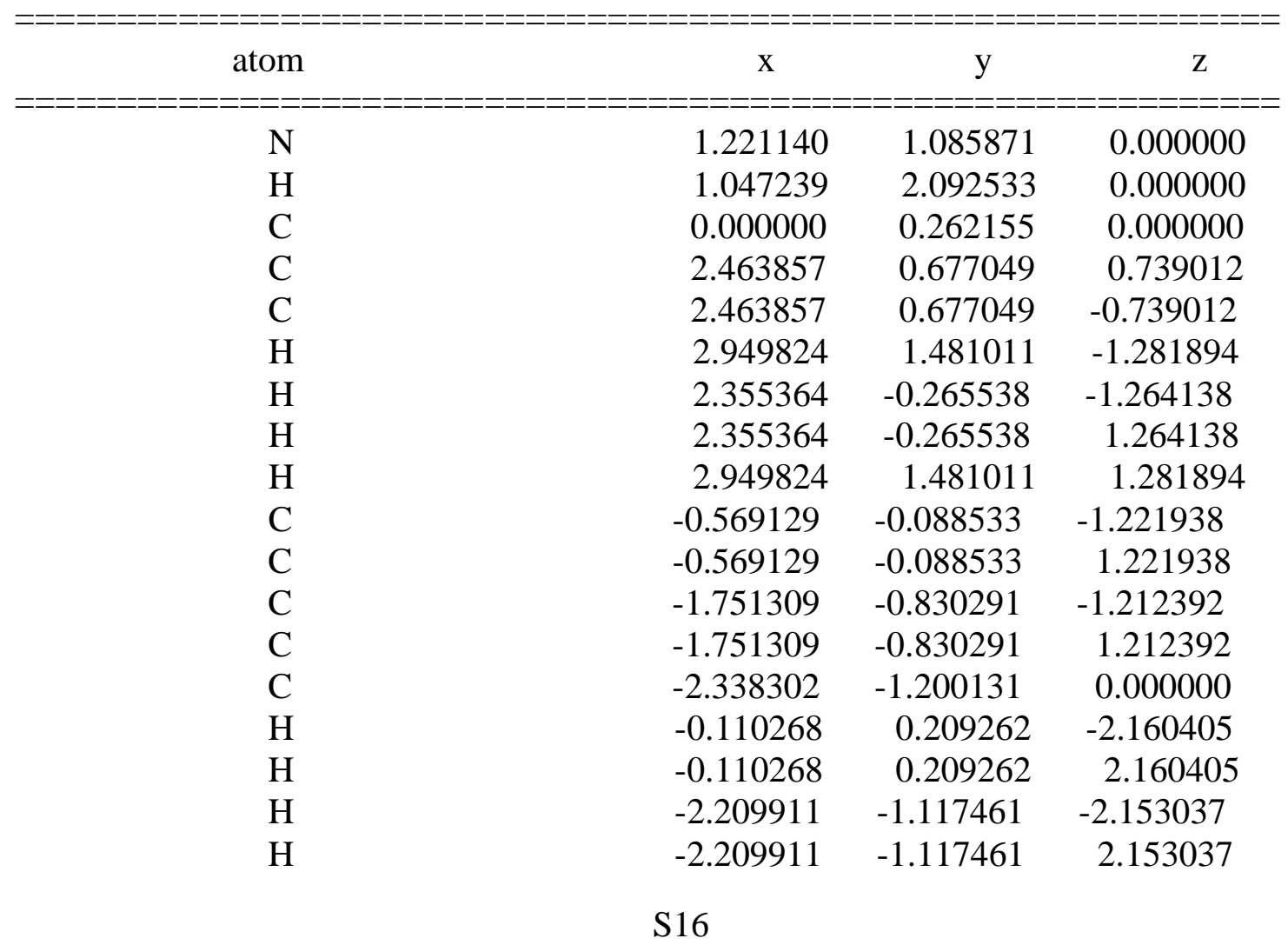


$\mathrm{H} \quad-3.256446 \quad-1.779027 \quad 0.000000$

Table S25. The Coordinates of Atoms of N-Protonated 10

Total Energy (au):-404.671295435(B3LYP/6-31G*)

\begin{tabular}{|c|c|c|c|}
\hline atom & $\mathrm{x}$ & $\mathrm{y}$ & $\mathrm{z}$ \\
\hline $\mathrm{N}$ & -1.141250 & -0.589770 & 0.000000 \\
\hline $\mathrm{H}$ & -2.015359 & -0.052246 & 0.000000 \\
\hline $\mathrm{C}$ & 0.000000 & 0.356244 & 0.000000 \\
\hline $\mathrm{C}$ & -1.247107 & -1.692455 & 1.070733 \\
\hline $\mathrm{C}$ & -1.247107 & -1.692455 & -1.070733 \\
\hline $\mathrm{C}$ & -1.773678 & -2.668417 & 0.000000 \\
\hline $\mathrm{H}$ & -0.238142 & -1.915613 & 1.418176 \\
\hline $\mathrm{H}$ & -1.891104 & -1.411383 & 1.904288 \\
\hline $\mathrm{H}$ & -0.238142 & -1.915613 & -1.418176 \\
\hline $\mathrm{H}$ & -1.891104 & -1.411383 & -1.904288 \\
\hline $\mathrm{H}$ & -1.329617 & -3.663788 & 0.000000 \\
\hline $\mathrm{H}$ & -2.862189 & -2.757681 & 0.000000 \\
\hline $\mathrm{C}$ & 0.501117 & 0.805489 & 1.219587 \\
\hline $\mathrm{C}$ & 0.501117 & 0.805489 & -1.219587 \\
\hline $\mathrm{C}$ & 1.550936 & 1.725434 & 1.210564 \\
\hline $\mathrm{C}$ & 1.550936 & 1.725434 & -1.210564 \\
\hline $\mathrm{C}$ & 2.075316 & 2.182353 & 0.000000 \\
\hline $\mathrm{H}$ & 0.090211 & 0.457184 & 2.162601 \\
\hline $\mathrm{H}$ & 0.090211 & 0.457184 & -2.162601 \\
\hline $\mathrm{H}$ & 1.955770 & 2.081659 & 2.152298 \\
\hline $\mathrm{H}$ & 1.955770 & 2.081659 & -2.152298 \\
\hline $\mathrm{H}$ & 2.893270 & 2.895718 & 0.000000 \\
\hline
\end{tabular}

Table S26 The Coordinates of Atoms of N-Protonated 11e Total Energy (au):-444.012598219(B3LYP/6-31G*)

\begin{tabular}{|c|c|c|c|}
\hline atom & $\mathrm{X}$ & $\mathrm{y}$ & $\mathrm{Z}$ \\
\hline ="="="="="="="=" & $E=======$ & $=========$ & $========$ \\
\hline $\mathrm{N}$ & 0.637005 & -0.718892 & 0.000000 \\
\hline $\mathrm{H}$ & 1.655053 & -0.580446 & 0.000000 \\
\hline $\mathrm{C}$ & 0.000000 & 0.637728 & 0.000000 \\
\hline $\mathrm{C}$ & 0.316434 & -1.612096 & 1.196302 \\
\hline $\mathrm{C}$ & 0.316434 & -1.612096 & -1.196302 \\
\hline $\mathrm{C}$ & 0.798051 & -3.006830 & 0.777591 \\
\hline $\mathrm{C}$ & 0.798051 & -3.006830 & -0.777591 \\
\hline $\mathrm{H}$ & 0.809987 & -1.216092 & 2.083364 \\
\hline
\end{tabular}


Supporting Information

\begin{tabular}{lccc}
$\mathrm{H}$ & -0.766071 & -1.553259 & 1.327388 \\
$\mathrm{H}$ & 0.809987 & -1.216092 & -2.083364 \\
$\mathrm{H}$ & -0.766071 & -1.553259 & -1.327388 \\
$\mathrm{H}$ & 1.800668 & -3.203890 & 1.166625 \\
$\mathrm{H}$ & 0.136541 & -3.769194 & 1.194739 \\
$\mathrm{H}$ & 1.800668 & -3.203890 & -1.166625 \\
$\mathrm{H}$ & 0.136541 & -3.769194 & -1.194739 \\
$\mathrm{C}$ & -0.264937 & 1.267436 & 1.216410 \\
$\mathrm{C}$ & -0.264937 & 1.267436 & -1.216410 \\
$\mathrm{C}$ & -0.825604 & 2.545528 & 1.207448 \\
$\mathrm{C}$ & -0.825604 & 2.545528 & -1.207448 \\
$\mathrm{C}$ & -1.107862 & 3.184526 & 0.000000 \\
$\mathrm{H}$ & -0.047454 & 0.791517 & 2.165441 \\
$\mathrm{H}$ & -0.047454 & 0.791517 & -2.165441 \\
$\mathrm{H}$ & -1.038643 & 3.037177 & 2.151186 \\
$\mathrm{H}$ & -1.038643 & 3.037177 & -2.151186 \\
$\mathrm{H}$ & -1.544300 & 4.178209 & 0.000000 \\
\hline$=========================================$
\end{tabular}

Table S27. The Coordinates of Atoms in N-Protonated 12p Total Energy (au):-483.330984902(B3LYP/6-31G*)

\begin{tabular}{|c|c|c|c|}
\hline atom & $\mathrm{x}$ & $\mathrm{y}$ & $\mathrm{z}$ \\
\hline $\mathrm{N}$ & 0.000000 & 0.676111 & 0.000000 \\
\hline $\mathrm{H}$ & -0.766529 & 1.361052 & 0.000000 \\
\hline $\mathrm{C}$ & -0.640989 & -0.698417 & 0.000000 \\
\hline $\mathrm{C}$ & 0.831105 & 0.970945 & 1.251033 \\
\hline $\mathrm{C}$ & 0.831105 & 0.970945 & -1.251033 \\
\hline $\mathrm{C}$ & 1.383285 & 2.394605 & 1.248352 \\
\hline $\mathrm{C}$ & 1.383285 & 2.394605 & -1.248352 \\
\hline $\mathrm{C}$ & 2.216664 & 2.692065 & 0.000000 \\
\hline $\mathrm{C}$ & -0.962159 & -1.313593 & 1.212373 \\
\hline $\mathrm{C}$ & -0.962159 & -1.313593 & -1.212373 \\
\hline $\mathrm{C}$ & -1.590367 & -2.560038 & 1.203809 \\
\hline $\mathrm{C}$ & -1.590367 & -2.560038 & -1.203809 \\
\hline $\mathrm{C}$ & -1.903825 & -3.188322 & 0.000000 \\
\hline $\mathrm{H}$ & 1.620138 & 0.214182 & 1.263396 \\
\hline $\mathrm{H}$ & 0.183696 & 0.821167 & 2.112397 \\
\hline $\mathrm{H}$ & 1.620138 & 0.214182 & -1.263396 \\
\hline $\mathrm{H}$ & 0.183696 & 0.821167 & -2.112397 \\
\hline $\mathrm{H}$ & 1.976367 & 2.508657 & 2.162326 \\
\hline $\mathrm{H}$ & 0.552278 & 3.109452 & 1.330633 \\
\hline $\mathrm{H}$ & 1.976367 & 2.508657 & -2.162326 \\
\hline $\mathrm{H}$ & 0.552278 & 3.109452 & -1.330633 \\
\hline
\end{tabular}


Supporting Information

$\begin{array}{lrrc}\mathrm{H} & 3.125021 & 2.075276 & 0.000000 \\ \mathrm{H} & 2.541737 & 3.736584 & 0.000000 \\ \mathrm{H} & -0.741683 & -0.861139 & 2.169858 \\ \mathrm{H} & -0.741683 & -0.861139 & -2.169858 \\ \mathrm{H} & -1.832310 & -3.033524 & 2.149909 \\ \mathrm{H} & -1.832310 & -3.033524 & -2.149909 \\ \mathrm{H} & -2.390667 & -4.158266 & 0.000000 \\ =======================================\end{array}$

Table S28. The Coordinates of Atoms of N-Protonated 12v Total Energy (au):-483.340031618(B3LYP/6-31G*)

\begin{tabular}{|c|c|c|c|}
\hline atom & $\mathrm{x}$ & $\mathrm{y}$ & $\mathrm{Z}$ \\
\hline $\mathrm{N}$ & 0.000000 & 0.654969 & 0.000000 \\
\hline $\mathrm{H}$ & -0.782071 & 1.319940 & 0.000000 \\
\hline $\mathrm{C}$ & -0.610649 & -0.700565 & 0.000000 \\
\hline $\mathrm{C}$ & 0.805201 & 0.956934 & 1.263406 \\
\hline $\mathrm{C}$ & 0.805201 & 0.956934 & -1.263406 \\
\hline $\mathrm{C}$ & 1.278568 & 2.408482 & 1.263133 \\
\hline $\mathrm{C}$ & 1.278568 & 2.408482 & -1.263133 \\
\hline $\mathrm{C}$ & 2.084142 & 2.744786 & 0.000000 \\
\hline $\mathrm{C}$ & -2.000216 & -0.791682 & 0.000000 \\
\hline $\mathrm{C}$ & -2.593498 & -2.055136 & 0.000000 \\
\hline $\mathrm{C}$ & -1.796934 & -3.200941 & 0.000000 \\
\hline $\mathrm{C}$ & -0.404662 & -3.088781 & 0.000000 \\
\hline $\mathrm{C}$ & 0.202598 & -1.832480 & 0.000000 \\
\hline $\mathrm{H}$ & 1.642196 & 0.254641 & 1.263767 \\
\hline $\mathrm{H}$ & 0.151696 & 0.720130 & 2.105947 \\
\hline $\mathrm{H}$ & 1.642196 & 0.254641 & -1.263767 \\
\hline $\mathrm{H}$ & 0.151696 & 0.720130 & -2.105947 \\
\hline $\mathrm{H}$ & 1.879035 & 2.562102 & 2.166165 \\
\hline $\mathrm{H}$ & 0.411534 & 3.078239 & 1.352535 \\
\hline $\mathrm{H}$ & 1.879035 & 2.562102 & -2.166165 \\
\hline $\mathrm{H}$ & 0.411534 & 3.078239 & -1.352535 \\
\hline $\mathrm{H}$ & 3.024645 & 2.177974 & 0.000000 \\
\hline $\mathrm{H}$ & 2.355057 & 3.804665 & 0.000000 \\
\hline $\mathrm{H}$ & -2.621364 & 0.101908 & 0.000000 \\
\hline $\mathrm{H}$ & -3.675483 & -2.138012 & 0.000000 \\
\hline $\mathrm{H}$ & -2.260368 & -4.182352 & 0.000000 \\
\hline $\mathrm{H}$ & 0.215264 & -3.979524 & 0.000000 \\
\hline $\mathrm{H}$ & 1.285477 & -1.755796 & 0.000000 \\
\hline
\end{tabular}




\section{Table S29}

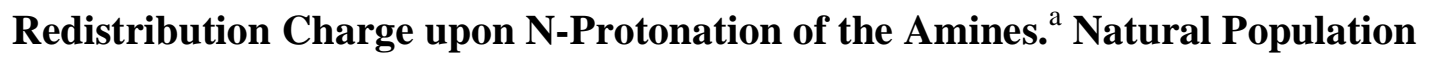
Analysis (B3LYP/6-31G* level) of the N-H atoms of the N-protonataed Amines and the N Atom of the Neutral Amine based on B3LYP6-31G* Optimized Structures.

\begin{tabular}{|c|c|c|c|c|}
\hline Ion & $\mathrm{N}-\mathrm{H}$ charge $^{\mathrm{b}}$ & Amine & $\mathrm{N}$ charge & Charge Retention $^{\mathrm{a}}$ \\
\hline $1 \mathrm{H}$ & -0.152 & 1 & -0.656 & +0.504 \\
\hline $2 \mathbf{H}$ & -0.168 & 2 & -0.698 & +0.530 \\
\hline $\mathbf{3 H}$ & -0.156 & 3 & -0.697 & +0.541 \\
\hline $4 H$ & -0.155 & 4 & -0.700 & +0.545 \\
\hline $5 H$ & +0.023 & 5 & -0.474 & +0.497 \\
\hline $6 H$ & +0.004 & 6 & -0.506 & +0.510 \\
\hline $7 \mathbf{H}$ & +0.011 & 7 & -0.504 & +0.515 \\
\hline $8 H$ & +0.012 & 8 & -0.507 & +0.519 \\
\hline 9pH & +0.028 & $9 p$ & -0.451 & $+0.479^{\mathrm{c}}$ \\
\hline 9vH & +0.035 & $9 \mathbf{v}$ & -0.487 & +0.522 \\
\hline 10vH & +0.008 & $10 p$ & -0.459 & $+0.467^{\mathrm{c}}$ \\
\hline $10 \mathrm{vH}$ & +0.008 & $10 \mathrm{v}$ & -0.531 & +0.539 \\
\hline 10pH & +0.013 & $10 p$ & -0.459 & +0.472 \\
\hline 11vH & +0.013 & 11hc & -0.453 & $+0.466^{\mathrm{c}}$ \\
\hline 11vH & +0.013 & $11 v$ & -0.531 & +0.544 \\
\hline 11eH & +0.020 & 11e & -0.457 & +0.477 \\
\hline $12 \mathrm{vH}$ & +0.014 & $12 t w$ & -0.487 & $+0.501^{\mathrm{c}}$ \\
\hline $12 \mathrm{vH}$ & +0.014 & $12 v$ & -0.533 & +0.547 \\
\hline 12pH & +0.019 & $12 p$ & -0.465 & +0.484 \\
\hline
\end{tabular}

a This value indicates the magnitude of the charge which is accumulated within the $\mathrm{NH}$ moiety of the ion upon N-protonation.

b. Summation of charges of the $\mathrm{N}$ and (N)-H atoms.

c. Protonation process of N-phenyl amines, shown in Table 1 
Table S30 The pyramidalization angle $\left(\beta_{1}, \beta_{2}\right.$, and $\beta_{3}$.see Scheme 1$)$ of inner and outer components of the RHO of the amine nitrogen atom (which represents the reactive lonepair electrons)

\begin{tabular}{|c|c|c|c|c|c|c|c|c|c|}
\hline \multirow[b]{2}{*}{ amines } & \multicolumn{4}{|c|}{ inner $\mathrm{p}^{a}$} & \multicolumn{4}{|c|}{ outer $\mathrm{p}^{b}$} & \multirow[b]{2}{*}{$\beta^{c}$} \\
\hline & $\beta_{1}$ & $\beta_{2}$ & $\beta_{3}$ & average & $\beta 1$ & $\beta 2$ & $\beta 3$ & average & \\
\hline 1 & 122.79 & 118.91 & 118.91 & 120.20 & 120.16 & 121.05 & 121.05 & 120.75 & 120.65 \\
\hline 2 & 111.96 & 111.18 & 111.18 & 111.44 & 110.00 & 112.44 & 112.44 & 111.63 & 111.49 \\
\hline 3 & 110.28 & 108.85 & 108.85 & 109.33 & 108.53 & 109.80 & 109.80 & 109.38 & 109.35 \\
\hline 4 & 109.05 & 108.02 & 108.02 & 108.36 & 107.09 & 108.97 & 108.97 & 108.34 & 108.36 \\
\hline 5 & 117.90 & 116.50 & 116.50 & 116.96 & 117.64 & 116.71 & 116.71 & 117.02 & 117.13 \\
\hline 6 & 108.91 & 109.47 & 109.47 & 109.29 & 109.10 & 109.35 & 109.35 & 109.27 & 109.25 \\
\hline 7 & 107.78 & 107.68 & 107.68 & 107.71 & 108.02 & 107.54 & 107.54 & 107.70 & 107.71 \\
\hline 8 & 107.18 & 107.33 & 107.33 & 107.28 & 107.41 & 107.22 & 107.22 & 107.28 & 107.28 \\
\hline $9 p$ & 115.07 & 113.99 & 113.99 & 114.35 & 114.33 & 114.60 & 114.60 & 114.51 & 114.48 \\
\hline $9 v$ & 116.36 & 115.47 & 115.47 & 115.77 & 119.96 & 112.46 & 112.46 & 114.96 & 115.87 \\
\hline $10 p$ & 102.50 & 102.93 & 102.93 & 102.78 & 103.63 & 102.18 & 102.18 & 102.66 & 102.76 \\
\hline $10 v$ & 106.95 & 107.92 & 107.92 & 107.60 & 109.33 & 106.33 & 106.33 & 107.33 & 107.53 \\
\hline $11 \mathrm{e}$ & 100.47 & 99.94 & 99.94 & 100.12 & 101.89 & 99.15 & 99.15 & 100.06 & 100.13 \\
\hline 11hc & 90.35 & 90.07 & 90.63 & 90.35 & 90.37 & 88.95 & 91.74 & 90.35 & 90.35 \\
\hline $11 v$ & 105.46 & 106.20 & 106.20 & 105.96 & 106.71 & 105.50 & 105.50 & 105.90 & 105.94 \\
\hline $12 \mathrm{tw}$ & 102.08 & 102.41 & 102.47 & 102.32 & 103.05 & 101.99 & 101.87 & 102.30 & 102.32 \\
\hline $12 p$ & 101.31 & 101.27 & 101.27 & 101.28 & 102.95 & 100.42 & 100.42 & 101.26 & 101.28 \\
\hline $12 \mathrm{v}$ & 104.78 & 105.89 & 105.89 & 105.52 & 105.57 & 105.49 & 105.49 & 105.51 & 105.51 \\
\hline
\end{tabular}

a. $\quad \beta 1, \beta 2$, and $\beta 3$ were defined as the angles formed by the inner $\mathrm{p}$ AO components (6$31 \mathrm{G}^{*}$ ) of RHO and the $\mathrm{N}-\mathrm{X}$ vectors, where $\mathrm{X}$ is an atom adjacent to $\mathrm{N}$ (Scheme 1).

b. $\quad \beta 1, \beta 2$, and $\beta 3$ were defined as the angles formed by the outer $\mathrm{p}$ AO components (6$31 \mathrm{G}^{*}$ ) of RHO and the $\mathrm{N}-\mathrm{X}$ vectors, where $\mathrm{X}$ is an atom adjacent to $\mathrm{N}$ (Scheme 1).

c. $\quad \beta$ is the angle formed by $\mathrm{N}-\mathrm{X}$ and a vector originating from $\mathrm{N}$, which is determined so that it makes three equivalent angles with $\mathrm{N}-\mathrm{X}$. 
Table S31 The average values of $\beta_{1}, \beta_{2}$, and $\beta_{3}$ of the inner and outer $\mathrm{p}$-AO components of the RHOs of N,N-dimethylamine upon changes of the bond angle $(\alpha)$. Comparison with the $\beta$ values. ${ }^{\mathrm{a}}$

(a) Inner $\mathrm{p}-\mathrm{AO}$ components of the $\mathrm{RHO}$

\begin{tabular}{|c|c|c|c|c|c|}
\hline$\alpha$ & $\beta$ & $\beta 1$ & $\beta 2$ & $\beta 3$ & average \\
\hline 60.5 & $108.6^{b}$ & 115.3 & 109.1 & 102.9 & 107.0 \\
\hline 90.4 & $108.6^{b}$ & 109.9 & 107.7 & 107.7 & 108.5 \\
\hline 104.8 & $108.6^{b}$ & 109.9 & 108.2 & 108.2 & 108.6 \\
\hline 112.1 & $108.6^{b}$ & 109.4 & 108.2 & 108.2 & 108.6 \\
\hline $112.8^{c}$ & 120.7 & 120.4 & 120.7 & 120.7 & 120.6 \\
\hline $112.8^{c}$ & 111.5 & 112.3 & 111.1 & 111.1 & 111.5 \\
\hline $112.8^{c}$ & 109.4 & 110.1 & 109.0 & 109.0 & 109.4 \\
\hline $112.8^{c}$ & 108.4 & 109.1 & 108.0 & 108.0 & 108.4 \\
\hline $112.8^{d}$ & $108.6^{d}$ & 109.3 & 108.3 & 108.3 & 108.6 \\
\hline \multicolumn{6}{|c|}{ (b) Outer p-AO components of the RHO } \\
\hline$\alpha$ & $\beta$ & $\beta 1$ & $\beta 2$ & $\beta 3$ & average \\
\hline 60.5 & $108.6^{b}$ & 111.4 & 106.2 & 106.2 & 108.0 \\
\hline 90.4 & $108.6^{b}$ & 106.9 & 109.7 & 109.7 & 108.8 \\
\hline 104.8 & $108.6^{b}$ & 107.1 & 109.5 & 109.5 & 108.7 \\
\hline 112.1 & $108.6^{b}$ & 107.3 & 109.3 & 109.3 & 108.6 \\
\hline $112.8^{c}$ & 120.7 & 119.2 & 121.0 & 121.0 & 120.4 \\
\hline $112.8^{c}$ & 111.5 & 110.4 & 112.0 & 112.0 & 111.4 \\
\hline $112.8^{c}$ & 109.4 & 108.1 & 110.0 & 110.0 & 109.3 \\
\hline $112.8^{c}$ & 108.4 & 107.0 & 109.0 & 109.0 & 108.3 \\
\hline $112.8^{d}$ & $108.6^{d}$ & 107.3 & 109.2 & 109.2 & 108.6 \\
\hline
\end{tabular}

$a$. Bond angle $\alpha$ and pyramidalization angle $\beta$ are shown in Scheme 1 .

$b$. This $\beta$ value is the optimized pyramidalization angle of $\mathrm{N}, \mathrm{N}$-dimethylamine.

$c$. This $\alpha$ value is obtained by structural optimization of $\mathrm{N}, \mathrm{N}$-dimethylamine.

$d$. The optimized structure of $\mathrm{N}, \mathrm{N}$-dimethylamine was used. 
Table S32 Summary of the Percentage s, p, and d Characters of the Nitrogen Atom Component of the RHO. ${ }^{a}$

\begin{tabular}{|c|c|c|c|}
\hline & $S$ & $p$ & $d$ \\
\hline 1 & 18.89 & 80.99 & 0.12 \\
\hline 2 & 9.95 & 89.99 & 0.06 \\
\hline 3 & 9.20 & 90.75 & 0.05 \\
\hline 4 & 8.71 & 91.25 & 0.04 \\
\hline 5 & 16.24 & 83.65 & 0.11 \\
\hline 6 & 8.71 & 91.24 & 0.05 \\
\hline 7 & 8.25 & 91.71 & 0.04 \\
\hline 8 & 8.17 & 91.80 & 0.04 \\
\hline $9 p$ & 13.94 & 85.94 & 0.11 \\
\hline $9 v$ & 15.61 & 84.29 & 0.10 \\
\hline $10 p$ & 3.82 & 96.15 & 0.03 \\
\hline $10 v$ & 7.50 & 92.45 & 0.05 \\
\hline 11e & 2.99 & 97.00 & 0.02 \\
\hline 11hc & 0.00 & 100.00 & 0.00 \\
\hline $11 v$ & 7.15 & 92.81 & 0.03 \\
\hline $12 t w$ & 4.55 & 95.43 & 0.02 \\
\hline $12 p$ & 3.72 & 96.26 & 0.02 \\
\hline $12 v$ & 6.99 & 92.98 & 0.03 \\
\hline
\end{tabular}

a. After expanding an RHO by orthogonal natural atomic orbitals (NAOs), s, p, and d ratios were calculated by the squared sums of the coefficients for the $s, p$, and dype NAOs, respectively. 